\title{
Association of sarcoidosis, low-grade B-lymphoma and epidermoid carcinoma
}

\author{
J Romero, S Ramón y Cajal, JA Vargas, R Pérez-Maestu, C Salas, CA Regueiro, \\ G Aragon
}

\begin{abstract}
Summary
We report on a case of the so-called sarcoidosis-lymphoma syndrome in a 65-year-old man diagnosed as having sarcoidosis and, four years later, neurosarcoidosis. The diagnoses of epidermoid carcinoma of the skin and of stage IV monocytoid, small cell lymphocytic lymphoma were made five and seven years, respectively, after the initial diagnosis of sarcoidosis. It has been suggested that the increased mitotic activity of lymphocytes observed in sarcoidosis, favours their malignant transformation. Hypothetically, sarcoidosis might also influence the development of epidermoid carcinomas by depletion of circulating $\mathrm{T} 4 \mathrm{lymphocytes}$ and decreased resistance to oncogenic viruses that could lead to decreased tumour rejection in the epithelia exposed to carcinogenic stimuli.
\end{abstract}

Keywords: sarcoidosis-lymphoma syndrome, sarcoidosis, lymphoma

An association between sarcoidosis and malignant disease has been advocated by some authors. ${ }^{1}$ Lymphoma is the most frequently associated tumour, with an incidence 11 times greater than expected in patients with sarcoidosis. $^{2}$ This frequent association led to the description of the so-called sarcoidosis-lymphoma syndrome. ${ }^{3}$ While some authors have denied the existence of such a syndrome, ${ }^{4,5}$ the description of new cases ${ }^{6-8}$ suggests a nonrandom association between the two entities.

Puerta de Hierro

Hospital, $S$ Martín

de Porres 4, 28035-

Madrid, Spain

Department of

Radiation Oncology

J Romero

CA Regueiro

A de la Cruz

Department of

Pathology

S Ramón y Cajal

C Salas

Department of

Internal Medicine

JA Vargas

R Pérez-Maestu

Accepted 27 November 1996

\section{Case report}

In May 1988, a 58-year-old man was admitted for a planned surgical intervention of a nasal polyposis. Pre-operative routine chest X-ray showed bilateral hilar enlargement and rightsided paratracheal lymphadenopathy (figure 1). Physical examination revealed bilateral, mobile, supraclavicular, axillary and inguinal enlarged lymph nodes without hepatosplenomegaly. Full blood counts were normal and the erythrocyte sedimentation rate was $102 \mathrm{~mm} / \mathrm{h}$. Serum calcium, lactate dehydrogenase and serum electrophoresis were unremarkable. Angiotensin-converting enzyme (ACE) was not determined. Abdominal computed tomography (CT) scan revealed retroperitoneal and inguinal lymphadenopathy and mild hepatomegaly. Gallium scintigraphy showed increased left-sided mediastinal uptake. Bone marrow biopsy was negative. Bronchoscopy and transbronchial biopsy showed normal macroscopic findings, peri-alveolar fibrosis and alveolar macrophages. Histologic examination of a biopsied supraclavicular lymph node revealed non-caseating epithelioid granulomas with multinucleated giant cells negative to stain for acidalcohol resistant bacilli and fungi. A diagnosis of sarcoidosis was established and the patient was kept under observation.

In June 1992 the patient was admitted for progressive walking difficulties, lasting three days. Neurological examination disclosed weakness (4/5 strength) and claudication (Mingazinni sign) of both lower limbs. Sensory testing, deep-tendon reflexes and plantar response were normal. Laboratory tests, ACE, chest X-ray and gallium scintigraphy showed no alterations. Magnetic resonance imaging (MRI) of the spinal cord showed signal enhancement at T12-L1 level compatible with sarcoidal transverse myelitis. Steroid therapy was instituted with resolution of symptoms.

In June1993, nasal skin epidermoid carcinoma was diagnosed, which needed to be treated surgically three times because of two

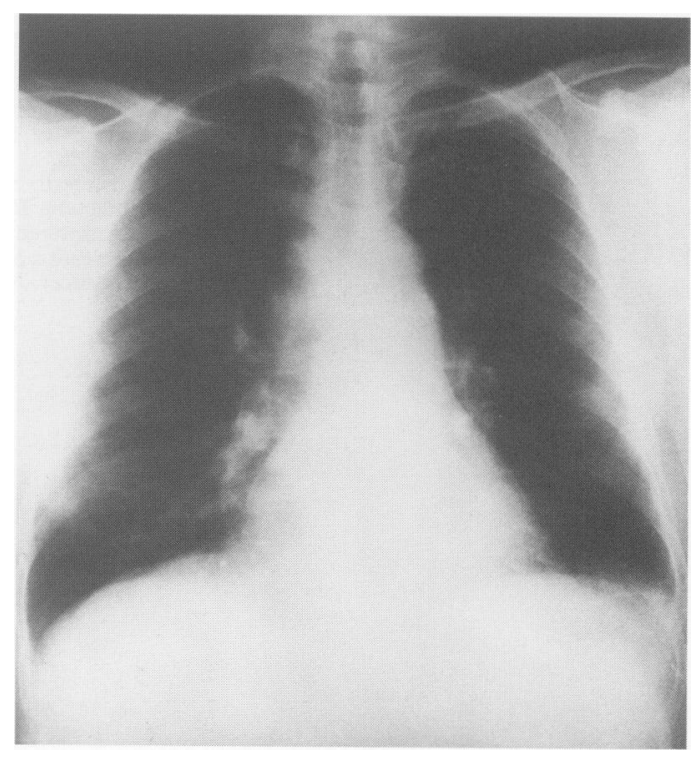

Figure 1 Chest X-ray showing bilateral hilar enlargement and right-sided paratracheal lymphadenopathy 
Figure 2 Histological section of the marginal zone B-lymphoma. Note that most of the cells are of medium size with abundant and pale cytoplasm (HE, original $x$ 450)

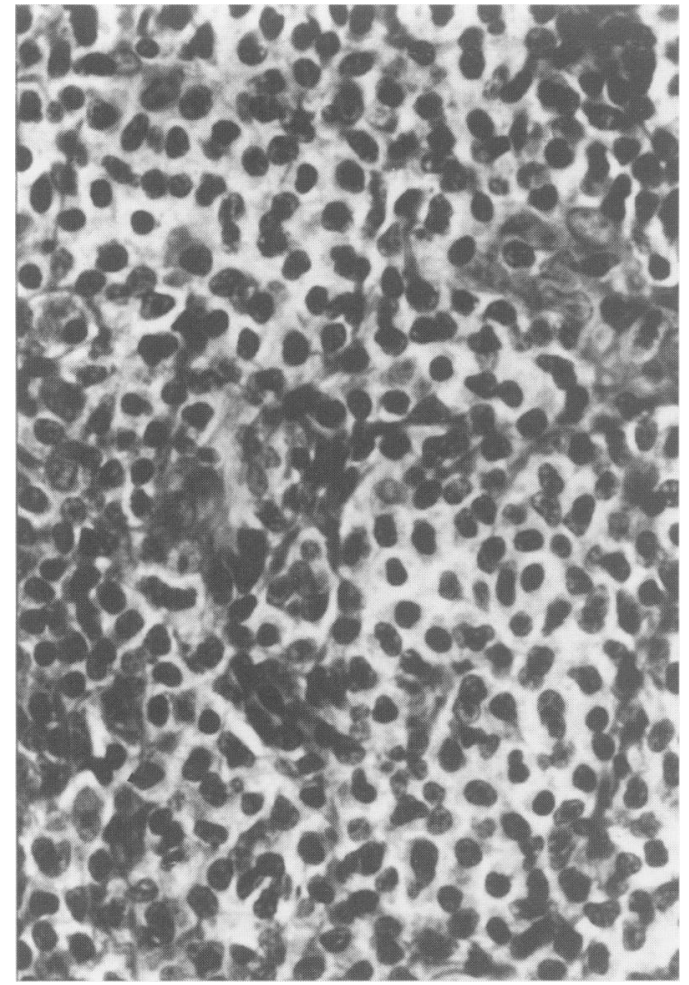

local relapses. In November 1994, after the third surgery, local radiotherapy was administered as there was tumoural invasion of surgical margin. One month after irradiation the patient presented with a right facial cranial nerve palsy. No other neurological abnormality was observed. Laboratory tests were normal. Chest X-ray denoted enlarged mediastinal lymph nodes. Bronchoalveolar lavage cell count showed polymorphonucleocytes $55 \%$, lymphocytes $20 \%$, macrophages $25 \%$. Abdomino-pelvic CT scan showed pathological retroperitoneal, pelvic and inguinal lymph nodes. Cranial CT scan, spinal MRI and cerebrospinal fluid were normal. The clinical picture was thought to be caused by neurosarcoidosis.

Five months after radiotherapy, the patient presented with an ulcerated cutaneous lesion located in the frontal region with positive biopsy for epidermoid carcinoma. Other findings from physical examination were bilateral cervical, supraclavicular, axillary and inguinal lymphadenopathy without hepatosplenomegaly. Blood count, serum chemistry, serum electrophoresis and ACE were unremarkable. Chest X-ray showed enlarged mediastinal lymph nodes and an interstitial pattern in the left inferior lobe. A biopsy of an axillary node demonstrated a lymphoid proliferation, diffuse pattern, consisting of small and intermediate cells with abundant and pale cytoplasm (figure 2 ). The pathological diagnosis was marginal zone B-lymphoma (REAL classification ${ }^{9}$ ) or small cell lymphocytic lymphoma (Working Formulation). The immunophenotype was: CD19+, CD20+, DR+, lambdat and CD5-. Thoraco-abdominal CT scan showed mediastinal, retroperitoneal, pelvic and inguinal lymphadenopathy. There was increased mediastinal and hilar uptake in the gallium scinti- graphy. The bone marrow was infiltrated by lymphoma. Surgical resection of the epidermoid carcinoma with skin graft was accomplished. As the lymphoma was asymptomatic, no further treatment was initiated at this time and the patient was followed-up at appropriate intervals

\section{Discussion}

Since a definitive diagnosis of sarcoidosis is often difficult to establish and local sarcoid reactions can be found in tumours, it has been suggested that patients with the sarcoidosislymphoma syndrome should meet various criteria (box 1). ${ }^{10}$ As a rule, sarcoidosis precedes lymphoma by several years, ${ }^{3,10}$ indicating a possible causal relationship. Typically, this syndrome presents in patients with a chronic active type of sarcoidosis, treated with corticosteroids, and who are, on average, 10 years older than the median age of other patients with sarcoidosis. ${ }^{3,10}$ These factors are associated with immunological abnormalities that are common in patients with sarcoidosis, such as a decreased numbers of circulating $T$ helper cells and hyperactivity of the B-lymphocytes, which contribute to the development of lymphoma.

Our patient presented in his late fifties with sarcoidosis involving various organs. He had received steroid treatment, and developed lymphoma seven years after the initial diagnosis of sarcoidosis. These are common features of the sarcoidosis-lymphoma syndrome. The patient also developed an epidermoid carcinoma. Although this association could be fortuitous, the existence of a second tumour of epithelial lineage has been previously reported in a large series ${ }^{3}$ in which four

\section{Sarcoidosis-lymphoma syndrome:} criteria

- each diagnosis should be separately and independently confirmed by biopsy from unrelated anatomical sites

- appropriate clinical, radiographic and biochemical features of each disease should be present; in particular, sarcoidosis should demonstrate multisystem involvement

- both diagnoses should be separated in time

Box 1

\section{Sarcoidosis-lymphoma syndrome:} features

- affected patients have a chronic active type of sarcoidosis of late onset

- in most cases, patients have received steroid treatment

- sarcoidosis precedes lymphoma by several years

- immunological abnormalities such as lymphopenia or anergy may be present 
out of 17 patients presented head and neck, breast, or cervical carcinomas in addition to lymphoma. Moreover, an increased incidence of lung cancer has been reported in patients with sarcoidosis. ${ }^{2}$

It has been suggested that the increased mitotic activity and dysregulation of lymphocytes observed in sarcoidosis, increases the risk of mutation. ${ }^{3}$ These factors, in addition to steroid treatment, may favour the development

1 Brincker $\mathrm{H}$. Coexistence of sarcoidosis and malignant disease: causality or coincidence? Sarcoidosis 1989; 61: 3143.

2 Brincker $H$, Wilbek $E$. The incidence of malignant tumours in patients with respiratory sarcoidosis. $\mathrm{Br} \mathcal{F}$ Cancer 1974; 29: 24751.

3 Brincker $\mathrm{H}$. The sarcoidosis-lymphoma syndrome. $\mathrm{Br} f$ Cancer 1986; 54: 46773.

Romer FK. Sarcoidosis and cancer. N Engl f Med 1982; 306: 1490.

5 McInerney PD. Sarcoidosis and lymphoma: is there an association? Postgrad Med $\mathcal{F} 1986 ; 62: 809$.

6 Masuda R, Toyoshima H, Bandou T, Isoyama T, Matsui Y, Takemura T. Malignant lymphoma of the stomach associated with systemic sarcoidosis. Cancer 1992; 70: 25926. of lymphoma. The pathogenic explanation for the existence of a second epithelial tumour is more complex. One could speculate that the depletion of circulating T4 lymphocytes and decreased resistance to oncogenic viruses might lead to decreased tumour rejection in the epithelia exposed to carcinogenic stimuli.

We thank Dr Miguel Yebra for a critical review of the manuscript.

7 Finke R, Lydtin H, Prechtel K. Sarcoidosis and immunocytoma. Am $\mathcal{F}$ Med 1986; 80: 93942.

8 Genestie C, Guettier C, Raphael M, et al. Sarcoidosis and non-Hodgkin lymphoma. A non-fortuitous association. Ann Pathol 1994; 14: 1203.

9 Harris NL, Jaffe ES, Stein $\mathrm{H}$, et al. A revised EuropeanAmerican classification of lymphoid neoplasms: a proposal from the International Lymphoma Study Group. Blood 1994; 84: 136192.

10 Brenan NJ, Fennelly J, Towers RP, Fitzgerald MX Sarcoidosis and lymphoma in the same patient. Postgrad Med f 1983; 59: 5815.

\title{
Renal cell carcinoma with solitary metastases appearing during 18 years of follow-up
}

\author{
M Siekierska-Hellmann, K Sworczak, A Lewczuk, K Błaut, E Boj
}

\begin{abstract}
Summary
We present a case of renal cell carcinoma with solitary metastases to the thyroid, the post-nephrectomy scar and the lungs, appearing during an 18-year period following nephrectomy.
\end{abstract}

Keywords: renal cell carcinoma, solitary metastases, diagnostic difficulties

Renal cell carcinoma is a disease with an elusive course. The absence of characteristic symptoms frequently delays the diagnosis. The classical 'diagnostic triad': haematuria, flank pain and abdominal mass, occurs only in 10$15 \%$ of patients, with $47 \%$ possibly having distant metastases at the time of diagnosis. ${ }^{1,2}$ Even with no metastases in $30 \%$ of patients, renal cell carcinoma presents multiple general symptoms such as fever, haematuria, polycythaemia, increased erythyrocyte sedimentation rate (ESR), weight loss, hypertension and hypercalcaemia. ${ }^{2}$ However, in $20 \%$ of cases renal cell carcinoma may have an asymptomatic course and may be discovered either incidentally or at autopsy. In 1-3\% the first symptoms appear as isolated distant metastases which, following surgery, give a $35 \%$ chance for a five-year survival. Solitary metastases of renal cell carcinoma to the lungs, ${ }^{3}$ pituitary, ${ }^{4}$ small intestine, ${ }^{5}$ thyroid, ${ }^{6,7}$ supraclavicular lymph nodes, neck region ${ }^{8}$ and skin $^{9,10}$ have been reported.

We present a patient with renal cell carcinoma with solitary metastases appearing during an 18-year period following nephrectomy.

\section{Case report}

A 49-year-old woman was admitted in 1974 for haematuria. Apart from that, she was asymptomatic and physical examination, laboratory tests, and chest X-ray were normal. ESR was $20 \mathrm{~mm} / \mathrm{h}$. Urography revealed an irregular lateral margin of the left kidney and displaced upper and lower calyces.

Renal arteriography confirmed the presence of a tumour in the middle part of the left kidney. In January 1975 nephrectomy was performed through the twelfth bed of the rib. The left kidney was excised with an orangesized tumour. No renal vessels invasion or the enlargement of surrounding lymph nodes were observed. Histopathological examination showed a tumour of $4 \times 4 \mathrm{~cm}$, the mass being yellowish-grey with blood extravasation. Microscopic examination demonstrated a renal cell carcinoma with no vessel invasion. She was discharged symptom-free and no complaints were reported in the following 10-year period. In March 1985 she noted a thyroid enlarge- 